\section{A pediatric case of Cardiobacterium hominis endocarditis}

\section{Priyanka Suresh, \\ R. Alexander Blackwood \\ Department of Pediatrics and \\ Communicable Diseases, University of Michigan Medical School, Ann Arbor, MI, USA}

\section{Abstract}

Gram negative endocarditis is relatively rare in pediatrics but when they occur they are most frequently caused by one of the HACEK (Haemophilus species, Actinobacillus actinomycetemcomitans, $C$. hominis, Eikenella corrodens and Kingella kingae) group of microorganisms. Within the HACEK group of microorganisms there have been approximately 100 cases of Cardiobacterium hominis endocarditis reported in the literature, but only 2 previous cases of endocarditis and one case of pericarditis have been reported in children. In this report, we present a case of a 12-year-old boy with a right ventricle to pulmonary artery conduit for Tetralogy of Fallot with pulmonary atresia who presented at an annual cardiology examination with a 3 week history of fatigue and was found to have a vegetation on routine echocardiogram. Subsequent blood cultures grew Cardiobacterium hominis and the patient was treated successfully with 6 weeks of appropriate antibiotic therapy. We present this case and a review of the literature of the HACEK group of microorganisms in pediatrics.

\section{Case Report}

A 12 year old boy, who was status post repair (shortly after birth) of Tetralogy of Fallot with pulmonary atresia and subsequent replacements of right ventricle to pulmonary artery conduit 4 years prior to current evaluation, was seen in an outside Pediatric cardiology clinic for his annual follow-up appointment. The appointment had been scheduled one month earlier on the patient's request, due to the onset of generalized fatigue and decreased endurance of 2-3 weeks duration. The patient denied symptoms of fever, chest pain, dizziness or syncope.

The patient had a history of poor dentition, poorly controlled asthma, sleep apnea, and allergic symptoms, which led to an adenoidectomy two years prior to current presentation.
The most recent right ventricle to pulmonary artery conduit replacement occurred 4 years prior to presentation which was complicated by Staphylococcus aureus mediastinitis. In clinic, by report, the physical examination revealed a well looking boy with thin habitus, in no acute distress with a heart rate of 102 beats/min, respiratory rate of 16 breaths/min and a blood pressure of $94 / 70 \mathrm{mmHg}$. Respiratory examination was significant for mild end expiratory wheezes. Cardiovascular examination demonstrated a regular rate and rhythm with a normal S1 and split fixed S2. A 3/6 graded harsh, early peaking, mid pitch systolic ejection murmur and a soft $2 / 4$ graded diastolic murmur were heard best at the left sternal border. Of note, there were no peripheral stigmata of endocarditis (splinter haemorrhages, Osler nodes or Janeway lesions) and the remainder of the physical examination was unremarkable. A routine echocardiogram showed a bright and thickened conduit valve with a suggestion of mass attached to the leaflet. This was not present on the previous echocardiogram (approximately 2 years prior).

Blood work at the clinic appointment was significant for a mildly elevated ESR of 25 $\mathrm{mm} /$ hour, CRP of $11.5 \mathrm{mg} / \mathrm{L}$ and a WBC of 5.92 cell $/ \mathrm{cm}^{3}$ with $16 \%$ of monocytes. Repeat blood work 2 days later showed slight improved CRP of $7.6 \mathrm{mg} / \mathrm{L}$, unchanged ESR and $\mathrm{CBC}$, normal electrolytes, LFT, CXR, and sinus films. A throat culture was positive for Streptococcus and ASO titre was elevated. The patient was started on amoxicillin for Streptococcal pharyngitis. At this time, the patient spiked a fever of $38.3^{\circ} \mathrm{C}$. The blood cultures taken both at presentation and two days later became positive for a slow growing gram negative rod.

Based on the positive blood cultures and the findings on the ECHO, the patient was referred for admission at University of Michigan C.S. Mott Children's Hospital with the diagnosis of subacute bacterial endocarditis four days after the initial clinic visit. Upon admission the patient was continued on amoxicillin for the Streptococcal pharyngitis and empirical treatment for bacterial endocarditis with intravenous (i.v.) vancomycin and i.v. piperacillin/tazobactam was initiated. Infectious Diseases was consulted three days after admission and the antibiotics were narrowed to i.v. ceftriaxone $1700 \mathrm{mg} \mathrm{q} 12$ hours since blood cultures had become negative after the first few doses of amoxicillin. At this time, oropharyngeal flora was suspected and organisms such as Kingella and Haemophilus species are not particularly virulent and are susceptible to ceftriaxone.

Aerobic and anaerobic blood cultures obtained on admission and on the subsequent day remained negative. A trans-thoracic echocardiogram on the second day after admission demonstrated evidence of a new
Correspondence: R. Alexander Blackwood, Department of Pediatrics and Communicable Diseases, University of Michigan Medical School, D5101 Medical Professional Building/SPC 5718, East Medical Center Drive 1500, Ann Arbor, 48109-5718, USA.

Tel. +1.734.763.2440 - Fax: +1.734.232.3859.

E-mail: rab@umich.edu

Key words: Cardiobacterium hominis, endocarditis, HACEK.

Conflict of interests: the authors declare no potential conflict of interests.

Received for publication: 30 November 2012.

Revision received: 8 February 2013

Accepted for publication: 8 March 2013.

This work is licensed under a Creative Commons Attribution NonCommercial 3.0 License (CC BYNC 3.0).

(C) Copyright P. Suresh and R.A. Blackwood et al., 2013

Licensee PAGEPress, Italy

Infectious Disease Reports 2013; 5:e7

doi:10.4081/idr.2013.e7

oscillating mass on a pulmonary valve leaflet concerning for a possible vegetation. The local laboratory was initially unable to identify the gram negative rod isolated from their clinic and the organism was sent to the University of Michigan microbiology laboratory where it was identified as Cardiobacterium hominis ( $C$. hominis) that was susceptible to amoxicillin/ clavulanate, meropenem, ceftriaxone and levofloxacin and resistant to ampicillin. The combined susceptibilities from the two laboratories are shown in Table 1.

Throughout the duration of admission, the patient remained afebrile and well-appearing and was discharged home on the fifth hospital day in stable condition on i.v. ceftriaxone therapy through a right upper extremity peripherally inserted central catheter line to complete a 6 week course of antibiotics. Two weeks into the course of treatment, the patient developed an intense pruritic rash near his peripherally inserted central catheter (PICC) site and along the PICC line track concerning for allergy. Ceftriaxone was discontinued and ampicillin/ sulbactam was started. However, the patient developed another lacy rash and ampicillin/sulbactam was then switched to levofloxacin 10 $\mathrm{mg} / \mathrm{kg} /$ day orally for the remaining two weeks of antibiotic therapy.

\section{Discussion}

C. hominis is a fastidious gram negative 
bacillus which is part of the normal oropharyngeal flora. ${ }^{1}$ It is a member of the HACEK (Haemophilus parainfluenzae, Haemophilus aphrophilus, and Haemophilus paraphrophilus, Actinobacillus actinomycetemcomitans, C. hominis, Eikenella corrodens, and Kingella kingae) group of microorganisms and is differentiated from them by a positive oxidase reaction and the production of indole. ${ }^{2}$ HACEK organisms account for between 3-10\% of the cases of native valve endocarditis but are the most common gram negative cause of endocarditis in children. ${ }^{3,4}$

The presentation of endocarditis with one of the HACEK microorganisms is often insidious in onset. Patients often experience malaise with or without low grade fevers which can last weeks to months before seeking medical attention or a diagnosis is confirmed. ${ }^{5}$ According to the Duke clinical criteria for the diagnosis of infective endocarditis, blood cultures should be incubated for at least two weeks. This allows time for slow growing bacteria like $C$. hominis and other HACEK microorganisms. ${ }^{6}$ Eighty-three percent of cases have also presented with constitutional symptoms such as fatigue. ${ }^{7}$ The patient presented here was afebrile prior to admission but had some intermittent fevers during the early phase of the admission. The patient therefore met modified Duke Criteria for definite infective endocarditis (echocardiographic findings, fever, predisposition, and positive blood cultures).

C. hominis is a slow growing, pleomorphic, gram negative bacillus. It is microaerophilic growing best with increased $\mathrm{CO}_{2}$ and $100 \%$ humidity and incubation may take up to 14 days although most cultures will become positive within 5 days. ${ }^{8}$ While $C$. hominis can be distinguished from other HACEK organisms by its characteristic fermentations pattern, glucose, maltose, mannitol, sorbitol, mannose and sucrose, the production of indole is a significant feature of the organism. In this case 3 days incubation was required for the gram negative bacilli to grow and it took 3 weeks for the organism to be identified as $C$. hominis. There have also been reports of cases where $C$. hominis could not be isolated from a standard blood culture. Mueller et al. describe a case of culture-negative endocarditis where $C$. hominis was diagnosed using broad-range polymerase chain reaction amplification of the 16 ribosomal RNA gene from arterial embolic tissue. They proposed the use of this technique to be beneficial as a supplement to the rapid, specific and sensitive identification of fastidious microorganisms. ${ }^{9}$

Initial isolates of $C$. hominis were penicillin and ampicillin sensitive. However, beta-lactamase producing strains of $C$. hominis have been identified and since antimicrobial susceptibility testing may be difficult to perform on HACEK microorganisms, ${ }^{6}$ the American Heart association now recommends that all HACEK microorganisms should be considered ampicillin resistant and third generation cephalosporins should be the treatment of choice. ${ }^{10}$ For patients who cannot tolerate beta lactams, trimethoprim/sulfamethoxazole, fluroquinolones and aztreonam are appropriate alternatives. ${ }^{6}$ This is the second case demonstrating beta-lactamase-producing $C$. hominis in a Pediatric patient. The first Pediatric case of beta-lactamase-producing $C$. hominis was described in a 7-year-old girl with a history of Tetralogy of Fallot who was treated with aztreonam. ${ }^{7}$ The initial plan in the case presented here was to treat the patient for a 6 -week course with Ceftriaxone. However, due to reactions concerning for an allergic response to ceftriaxone and ampicillin/sulbactam, levofloxacin was used to complete the 6-week course.

Underlying heart disease is the major risk factor for developing endocarditis with approximately $76 \%$ of cases occurring in patients with surgically repaired structural cardiac abnormalities. ${ }^{6,7}$ Because HACEK microorganisms are part of the normal oral flora, recent dental procedures are a risk factor for developing endocarditis with this group of microorganisms - especially for patients with underlying heart disease. Wormser et al. noted that 12 of 27 patients with endocarditis caused by one of the HACEK microorganisms had recent dental work or an oral infection prior to presentation. ${ }^{11}$ Additionally children with congenital heart disease harbor HACEK microorganisms to a greater extent and have more gingival inflammation than healthy children. ${ }^{12}$ In this case, the patient did not undergo a dental procedure but was noted to have poor dentition and developed pharyngitis with Group A Streptococcus which may have facilitated $C$. hominis entry.

\section{Conclusions}

The HACEK group of microorganisms can cause endocarditis in children, most commonly

Table 1. Antibiotic susceptibilities of $C$. hominis.

\begin{tabular}{lcc} 
Antibiotic & MilC value $(\mathrm{ug} / \mathrm{mL})$ & Interpretation \\
Ertapenem & 2 & Susceptible \\
Meropenem & $\leq 0.5$ & Susceptible \\
\hline Meropenem 2 & $\leq 0.25$ & Susceptible \\
Trimethoprim/sulfamethoxazole & $\leq 0.5$ & Susceptible \\
\hline Levofloxacin & $\leq 2$ & Susceptible \\
Moxifloxacin & $\leq 0.06$ & Susceptible \\
\hline Amoxicillin/clavulanate & $\leq 0.5 / 0.25$ & Susceptible \\
Ampicillin & 4 & Resistant \\
\hline Beta-lactamase & - & Positive
\end{tabular}

Table 2. A summary of pediatric case reports of $C$. hominis.

\begin{tabular}{|c|c|c|c|c|c|c|c|}
\hline Case & Age & Sex & Cardiac anomaly & Treatment & Complication & Surgery & Outcome \\
\hline 1 & 17 & M & Aortic valve & $\begin{array}{l}\text { Ceftriaxone } 6 \text { weeks/gent } \\
\text { and flucloxacillin } 2 \text { weeks }\end{array}$ & None & Valve replacement & Good \\
\hline 2 & 5 & M & $\begin{array}{c}\text { Tetralogy of Fallot } \\
\text { with pulmonary atresia }\end{array}$ & Ceftriaxone & None & Valve replacement & Good \\
\hline 3 & 10 & M & $\begin{array}{c}\text { Tetralogy of Fallot } \\
\text { with pulmonary atresia }\end{array}$ & $\begin{array}{c}\text { Ceftriaxone amp-sulbactam } \\
\text { and levofloxacin }\end{array}$ & None & None & Good \\
\hline Pericarditis & 10 & $\mathrm{~F}$ & None & Ceftriaxone & None & Partial pericardictomy & Good \\
\hline
\end{tabular}


with $K$. kingae and $H$. parainfluenzae. $C$. homin$i s$ is a rare cause of endocarditis in children and to our knowledge this is the third pediatric case reported. Additionally, there has been one reported case of $C$. hominis pericarditis in an otherwise healthy child (Table 2).

\section{References}

1. Slotnick IJ, Dougherty M. Further characterisation of an unclassified group of bacteria causing endocarditis in man: Cardiobacterium hominis gen. et sp.n. Antonie von Leeuwenhoek 1964;30:261-72.

2. Geraci JE, Greipp PR, Wilkowske CJ, et al. Cardiobacterium hominis endocarditis: four cases with clinical and laboratory observations. Mayo Clin Proc 1978;53:49-
53.

3. Mylonakis E, Calderwood SB. Infective endocarditis in adults. N Engl $\mathrm{J}$ Med 2001;345:1318-30.

4. Ferrieri P, Gewitz MH, Gerber MA et al. Unique featrues of infective endocarditis in childhood. Pediatrics 2002;109:931-43.

5. Currie PF, Codispoti M, Mankad PS, Godman MJ. Late aortic homograft valve endocarditis caused by Cardiobacterium hominis: a case report and review of the literature. Heart 2000;83:579-81.

6. Maurissen W, Eyskens B, Gewillig M, et al. Beta-lactamase-positive Cardiobacterium hominis strain causing endocarditis in a pediatric patient with Tetralogy of Fallot. Clin Microbiol News 2008;30:132-3.

7. Walkty A. Cardiobacterium endocarditis: a case report and review of literature. Can J Infect Dis Med Microbiol 2005;16:293-7.

8. Das M, Badley AD, Cockerill FR, et al.
Infective endocarditis caused by HACEK microorganisms. Annu Rev Med 1997;48: 25-33.

9. Mueller NJ, Kaplan V, Zbinden R, Altwegg M. Diagnosis of Cardiobacterium hominis endocarditis by broad-range PCR from arterio-embolic tissue. Infection 1999;27: 278-9.

10. Wilson WR, Karchmer AW, Dajani AS, et al. Antibiotic treatment of adults with infective endocarditis due to streptococci, enterococci, staphylococci, and HACEK microorganisms. JAMA 1995;21:1706-13.

11. Wormser GP, Bottone EJ. Cardiobacterium hominis: review of microbiologic and clinical features. Rev Infect Dis 1983;5:680-91.

12. Steelman R, Einzig S, Balian A, et al. Increased suseptibility to gingival colonization by specific HACEK microbes in children with congenital heart disease. J Clin Pediatr Dent 2000;25:91-4. 